\title{
Intraarticular Botulinum Toxin A for the Treatment of Painful Ankle Osteoarthritis-A Pilot Study
}

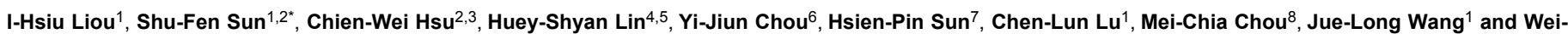
Hsuan Lee ${ }^{1}$

${ }^{1}$ Department of Physical Medicine and Rehabilitation, Kaohsiung Veterans General Hospital, Taiwan

${ }^{2}$ National Yang-Ming University School of Medicine, Taiwan

${ }^{3}$ Department of Internal Medicine, Kaohsiung Veterans General Hospital, Taiwan

${ }^{4}$ School of Nursing, Fooyin University, Taiwan

${ }^{5}$ Statistical Consultant of Research and Development, Department of Health, Kaohsiung City Government, Taiwan

${ }^{6}$ Department of Orthopedic Surgery, Kaohsiung Veterans General Hospital, Taiwan

${ }^{7}$ Department of General Surgery, Cheng Ching Hospital, Taiwan

${ }^{8}$ Department of Physical Medicine and Rehabilitation, Kaohsiung Veterans General Hospital-Pin-Tong Branch, Taiwan

*Corresponding author: Shu-Fen Sun, Department of Physical Medicine and Rehabilitation, Kaohsiung Veterans General Hospital, No 386, Ta-Chung 1st Road, Kaohsiung 813, Taiwan Tel: 886-73422121-4210; Fax: 886-73420243; E-mail: sfsun.tw@yahoo.com.tw

Received: February 11 2014, Accepted: May 01 2014, Pub date: May 102014

Copyright: @ 2014 , Sun SF, et al. This is an open-access article distributed under the terms of the Creative Commons Attribution License, which permits unrestricted use, distribution, and reproduction in any medium, provided the original author and source are credited.

\begin{abstract}
Purpose: Ankle osteoarthritis (OA) can cause substantial pain and functional limitations. Effective therapy has been a key therapeutic challenge. The aim of this pilot study is to investigate the effects of intra articular Botulinum toxin type A (BoNT-A) for the treatment of patients with painful ankle OA.

Methods: This was a prospective study conducted in a university-affiliated tertiary care medical center with 6month follow-up. Patients with painful ankle OA for at least 6 months and radio graphically verified as having stage II $\mathrm{OA}$ according to the Kellgren-Lawrence classification were enrolled. Eligible patients received single intraarticular injections of BoNT-A (100 units). The primary outcome was the Ankle Osteoarthritis Scale (AOS) score at 6-month follow-up. Secondary outcomes measures included American Orthopaedic Foot and Ankle Society (AOFAS) ankle/ hindfoot score, Visual analog scale (VAS) for ankle pain, single leg stance test (SLS), Timed "Up-and-Go" test (TUG) and consumption of rescue analgesics. Patients were evaluated at baseline, 2 weeks, 1 month, 3 months, and 6 months post injection. Patients' global satisfaction about therapy was assessed at 2 weeks, 1 month, 3 months, and 6 months post injection. Adverse events during the study period were recorded also.
\end{abstract}

Results: Thirty-six patients completed the study. All patients showed significant improvements in AOS, AOFAS, VAS, SLS, TUG scores and consumption of rescue analgesics at 2 weeks, 1, 3 and 6 months respectively after the injection $(p<0.001$ for each value compared with baseline). Patients' global satisfaction rate was high with no serious adverse events.

Conclusion: BoNT-A injection to the ankle joint is associated with improvement in pain, function and balance in patients with painful ankle OA. These effects can last for 6 months. This pilot study supports a possible role for BoNT-A as a treatment option for painful ankle OA. Future studies are needed to confirm the results.

Keywords: Ankle osteoarthritis; Botulinum toxin; Intraarticular injection

\section{Introduction}

Ankle osteoarthritis (OA) can cause pain and disability. Current treatment options include analgesics, non-steroidal anti-inflammatory medication (NSAIDs), weight loss, physical therapy, exercise, activity modification, assistive devices, local injections, and surgical treatment. The treatment goals are to reduce pain and improve function. Oral analgesics have substantial limitations because they may not provide sufficient joint pain relief and often produce intolerable drug side effects and adverse drug interactions [1,2]. Intra articular therapies with corticosteroids or viscosupplements may reduce pain, but the duration of effect is variable [3]. There is an increasing requirement for novel treatments of $\mathrm{OA}$ as the aging population is expanding with many patients who are unable to or prefer not to receive surgery. Effective and safe treatments that may reduce pain and improve function yet avoid the toxic effects of medications should be fully exploited. A potential treatment option for patients may be the intra articular application of Botulinum toxin type A (BoNT-A).

Preliminary evidence suggests that BoNT-A has a significant antinociceptive effect, when injected intra articularly, into painful joints [4-7]. These data suggest that BoNT-A may be a new therapeutic option for patients with OA. To date, there is rare published literature that evaluate the effects of intra articular BoNT-A in the treatment of ankle OA. The purpose of this study is to investigate the effects of intra articular BoNT-A for the treatment of patients with painful ankle OA. 
We hypothesize that intraarticular BoNT-A would result in statistically significant improvements in pain and function at 6 months.

\section{Subjects and Methods}

\section{Participants}

Patients in this study were referred from our outpatient orthopedic department with a diagnosis of ankle OA. All patients met the following inclusion criteria:(1) an age of 20-85 years, ankle pain that had lasted for at least 6 months, with no significant benefit from conservative treatment or with an inability to tolerate the side effects of medications; (2) ankle radiographs taken within 6 months equivalent to grade 2 on the Kellgren-Lawrence grading system [8]; (3) a current total Ankle Osteoarthritis Scale (AOS) score of (described below) of $>3$ and $<9$ (possible range, $0-10$ ); (4) a normal activity leveli.e., not bedridden or confined to a wheelchair, and are able to walk 30 meters without the aid of a walker, crutches or cane; and (5) no physical therapy, shoe changes or orthotic devices used during the study period.

Patients were excluded if they were women in pregnancy or lactation, had lower leg trauma other than ankle trauma, previous surgery involving the spine, hip or knee, the presence of an active joint infections of foot or ankle, previous surgery or arthroscopy on the ankle within 12 months, intra articular steroid or hyaluronate injection in the treated ankle in the preceding 6 months, or treatment with anticoagulants or immunosuppressive. Other exclusion criteria included a history of rheumatoid arthritis, gout, or any other inflammatory arthropathy, the presence of other comorbidity (such as diabetes mellitus, paresis, neoplasms or recent trauma) or poor health status that would interfere with the clinical assessments during the study.

Intake of analgesics or NSAIDs was not permitted during the study period, except that acetaminophen $(500 \mathrm{mg})$, limited to $4 \mathrm{~g}$ /day was allowed as rescue medication. If the treatment dose was above the stipulated limit (acetaminophen $4 \mathrm{~g} /$ day), the patient was regarded as a clinical failure. Patients taking analgesics or NSAIDs stopped them at least 7 days before the pre-injection assessment. Administration of acetaminophen was stopped at least 8 hours before the follow-up assessments. The administration of all analgesic medication during the study period was recorded on a diary card by the patient.

The study was approved by the institutional review board for human investigation and all subjects provided signed informed consent before being enrolled in the study.

\section{Botulinum Toxin and Injection Technique}

One hundred units of BoNT-A (Allergan, Inc, Irvine CA) were reconstituted in $2 \mathrm{ml}$ of normal saline. All patients received intraarticular BoNT-A injections by the same experienced physician using aseptic procedures. The ankle joint (tibiotalar) was injected by inserting the needle $1 \mathrm{~cm}$ anterior to the distal medial malleolus and advancing the needle posteriorly and slightly superiorly toward the middle of the ankle joint above the talus. If an effusion was present, it was aspirated before injecting.

\section{Outcome Assessments}

The clinical assessment included the following items:
(1) The AOS was a patient-rated, validated outcome measure that includes nine items on a pain subscale and nine items on a disability subscale [9]. Using the AOS, a score of 0 represented no pain or disability and 10 represented the worst pain or disability imaginable.

(2) AOFAS ankle/hindfoot score was a 100-point scale that devoted 40 points to pain, 50 points to function and 10 points to alignment [10]. The maximum score of 100 points denoted no pain and normal function and alignment.

(3) The patient rated the intensity of average ankle movement pain in the previous week using a $10-\mathrm{cm}$ horizontal Visual analog scale (VAS) [11]. The VAS was marked in 1-cm increments from "no pain" to "worst pain".

(4) Single-leg stance test (SLS) was done by raising one foot up without touching it to the supported lower extremity with ankle OA, keeping arms down at the sides, and maintained balance for as long as possible [12]. Failure occurs if the stance foot shift in any way or the non-stance foot touches the ground. Each participant performed 3 trials, and the best result was recorded.

(5) A timed "Up-and-Go" test (TUG) was used to measure functional mobility and dynamic balance of an individual [13]. A patient was asked to rise from an armchair, walk 3 meters at a safe and comfortable pace, turn around, walk back to the chair, and sit down again. The whole procedure was demonstrated first before the actual test. The time (in seconds) required to complete these tasks was recorded.

(6) Patients rated their global satisfaction level for ankle pain relief on weight bearing compared to their pre-injection condition at each follow-up visit. This rating was based on a 0-6 7-point categorical scale ranging from completely satisfied, satisfied, somewhat satisfied, no change, somewhat dissatisfied, dissatisfied to completely dissatisfied.

These outcome tests were conducted pre-injection and at 2 weeks, 1 month, 3 months, and 6 months post injection. Patients' global satisfaction was assessed at 2 weeks, 1 month, 3 months, and 6 months post injection. We chose several post injection measures to see the short-term ( 2 weeks, 1 month) and long term ( 3 months, 6 months) effects.

To monitor the safety of each injection, patients recorded any systemic and local adverse effects (defined as any unwanted effect whether it was thought to be related to the study or not) on a diary card.

\section{Statistical analysis}

All statistical procedures were conducted with the Statistical Package for the Social Sciences (version 12.0; SPSS Inc., Chicago, Illinois). The data were presented as the mean + standard deviation or frequency with percentage as appropriate. Change of outcome measures in AOS, AOFAS, VAS, SLS, TUG, rescue analgesics consumption and global satisfaction were assessed using repeated measures one-way analysis of variance (ANOVA). Furthermore, Bonferroni post hoc test for multiple comparisons were used to compare the differences of an outcome variable between any two time points if there was difference among measurement points. $\mathrm{P}$ values of less than 0.05 were regarded as significant. 
Page 3 of 5

\section{Results}

Thirty-eight patients met all eligibility criteria and participated for the BoNT-A injection study. Two patients withdrew from the study, one because of transportation problems and one due to fear of needle injection. A total of 36 patients (21 men and 15 women), mean age 48.9 years (age range 32-80 years), completed the 6-month follow-up. Ankle OA was attributed to primary OA without history of trauma or purulent arthritis in 10 patients. Secondary OA because of ligamentous injury, malleolar fracture, plafond fracture, talar fracture, previous purulent arthritis or other causes was noted in 26 patients. Table 1 summarized the demographic data and disease characteristics of these patients.

\begin{tabular}{|l|l|l|}
\hline Characteristic & Patients $(\mathbf{n}=\mathbf{3 6})$ & Range \\
\hline Age (years) & $48.9 \pm 10.4$ & $32-80$ \\
\hline Sex (M/F) & $21 / 15$ & \\
\hline Weight (kg) & $58.5 \pm 15.9$ & $52-78$ \\
\hline
\end{tabular}

\begin{tabular}{|l|l|l|}
\hline Height $(\mathbf{c m})$ & $166.4 \pm 6.6$ & $152-176$ \\
\hline $\begin{array}{l}\text { Etiology of osteoarthritis } \\
\text { (primary/secondary) }\end{array}$ & $26-\mathrm{Oct}$ & \\
\hline Heavy worker/light worker & $26-\mathrm{Oct}$ & \\
\hline Side of ankle injected (left/right) & $25-\mathrm{Nov}$ & \\
\hline Disease duration (years) & $5.6 \pm 3.8$ & $0.5-16$ \\
\hline
\end{tabular}

Table 1: Demographic data and disease characteristics of the patients, Data are mean \pm standard deviation or number

Table 2 provided a summary of outcome measures at each followup visit. Results of AOS, AOFAS, VAS, SLS, TUG tests and analgesics consumption improved significantly from baseline $(p<0.001$ at each time point compared with baseline) after completion of BoNT-A injections. The treatment effects were rapid at 2 weeks post injection and lasted for at least 6 months.

\begin{tabular}{|c|c|c|c|c|c|c|}
\hline Outcome & Baseline(A) & 2 week(B) & 1 month(C) & 3 months(D) & 6 months(E) & $\begin{array}{l}\text { Bonferroni post } \\
\text { hoc test }\end{array}$ \\
\hline Total AOSa & $4.9 \pm 1.6$ & $2.8 \pm 1.3$ & $2.5 \pm 1.4$ & $2.5 \pm 1.4$ & $2.7 \pm 1.2$ & $A B^{*} A C^{*} A D^{*} A E^{*}$ \\
\hline Pain subscalea & $4.5 \pm 1.3$ & $2.5 \pm 1.3$ & $2.3 \pm 1.4$ & $2.3 \pm 1.4$ & $2.4 \pm 1.2$ & $A B^{*} A C^{*} A D^{*} A E^{*}$ \\
\hline $\begin{array}{l}\text { Disability } \\
\text { subscalea }\end{array}$ & $5.2 \pm 1.9$ & $3.0 \pm 1.5$ & $2.8 \pm 1.6$ & $2.8 \pm 1.5$ & $2.9 \pm 1.3$ & $A B^{*} A C^{*} A D^{*} A E^{*}$ \\
\hline \multirow{2}{*}{$\begin{array}{l}\text { AOFAS ankle/ } \\
\text { hindfoot score }\end{array}$} & \multirow{2}{*}{$71.1 \pm 11.8$} & \multirow{2}{*}{$84.4 \pm 7.8$} & \multirow{2}{*}{$87.9 \pm 7.4$} & \multirow{2}{*}{$88.9 \pm 6.4$} & \multirow{2}{*}{$88.3 \pm 6.4$} & $A B^{*} A C^{*} A D^{*} A E^{*}$ \\
\hline & & & & & & $B C+B D \S$ \\
\hline VAS pain scalea & $4.0 \pm 1.8$ & $1.8 \pm 1.1$ & $1.7 \pm 1.2$ & $1.8 \pm 1.2$ & $1.8 \pm 0.9$ & $A B^{*} A C^{*} A D^{*} A E^{*}$ \\
\hline \multirow{2}{*}{ SLS (second) } & \multirow{2}{*}{$24.9 \pm 19.8$} & \multirow{2}{*}{$34.1 \pm 23.6$} & \multirow{2}{*}{$37.3 \pm 23.0$} & \multirow{2}{*}{$36.9 \pm 20.6$} & \multirow{2}{*}{$36.7 \pm 18.3$} & $A B^{*} A C^{*} A D^{*} A E^{*}$ \\
\hline & & & & & & $\mathrm{BC}+$ \\
\hline \multirow{2}{*}{ TUGa (second) } & \multirow{2}{*}{$8.4 \pm 3.0$} & \multirow{2}{*}{$7.2 \pm 2.2$} & \multirow{2}{*}{$6.4 \pm 1.6$} & \multirow{2}{*}{$6.5 \pm 1.5$} & \multirow{2}{*}{$6.7 \pm 1.7$} & $A B^{*} A C^{*} A D^{*} A E^{*}$ \\
\hline & & & & & & $\mathrm{BC}^{*} \mathrm{CE}+$ \\
\hline $\begin{array}{l}\text { Acetaminophen } \\
\text { (tablets/week) }\end{array}$ & $15.9 \pm 5.3$ & $8.4 \pm 4.1$ & $8.3 \pm 4.3$ & $8.8 \pm 4.9$ & $9.3 \pm 4.6$ & $A B^{*} A C^{*} A D^{*} A E^{*}$ \\
\hline
\end{tabular}

Table 2: Values are the mean \pm standard deviation; AOS=Ankle Osteoarthritis Scale, AOFAS=the American Orthopedic Foot and Ankle Society, $\mathrm{ROM}=$ range of motion SLS=single leg stance test; TUG=timed "Up-and-Go" test; the possible range for the AOS score was 0-10; the possible range for the AOFAS score was 0-100; a Higher scores represent worse pain or function; $+\mathrm{P}<0.05, \S \mathrm{P}<0.01,{ }^{\star} \mathrm{P}<0.001$

The improvement in the outcome variables attained during the study period had occurred by the time of the 2-week follow-up visit. Thereafter, the AOS and TUG scores trended downward (better), and the AOFAS Ankle-Hind foot and SLS scores trended upward (better) with time until 1-month follow-up. The maximal improvement in AOS, AOS pain subscales, TUG and SLS scores were at 1-month follow-up; whereas the maximal improvement in AOFAS Ankle-Hind foot scores were at 1-month and 3-month follow-up. The TUG scores then trended upward (poorer) at 3-month and 6-month follow-up. However, the entire outcome variables still remained significantly improved from baseline at 6-month follow-up $(\mathrm{p}<0.001)$.

The injection therapy resulted in a high rate of patient satisfaction. Most patients reported satisfaction at 2 week $(91.7 \%$, at least somewhat satisfied) follow-up visit (Table 3). At 6-month follow-up, 97.2\% of patients reported satisfaction (at least somewhat satisfied) about this therapy. No patient reported dissatisfaction or aggravation of the ankle symptoms compared with the baseline condition at any of the followup visits.

\begin{tabular}{|l|l|l|l|l|l|}
\hline & $\begin{array}{l}\text { Completely } \\
\text { Satisfied }\end{array}$ & $\begin{array}{l}\text { Satisfi } \\
\text { ed }\end{array}$ & $\begin{array}{l}\text { Somewha } \\
\text { t Satisfied }\end{array}$ & No Change & $\begin{array}{l}\text { Satisfaction } \\
\text { Rate }\end{array}$ \\
\hline 2 week & 5 & 17 & 11 & 3 & $91.70 \%$ \\
\hline 1 month & 6 & 20 & 8 & 2 & $94.40 \%$ \\
\hline 3 months & 9 & 12 & 15 & 0 & $100.00 \%$ \\
\hline
\end{tabular}


6 months 6

14

15

1

$97.20 \%$

Table 3: Numbers referred to number of patients who reported their level of global satisfaction when comparing the situation after injection with that before injection. No patients reported dissatisfaction throughout the study period.

All patients tolerated the treatment well. No severe or systemic adverse events were reported during the study period. Transient injection-site reactions with mild to moderate pain and local swelling were reported by 2 patients and these symptoms cleared in a few days.

\section{Discussion}

The results showed that injecting BoNT-A into the ankle joints was associated with pain reduction, function and balance improvement in patients with painful ankle OA. These effects could last for 6 months.

The results of this study using BoNT-A for painful ankle OA were consistent with those of previous studies using BoNT-A in the shoulder and knee joints $[6,14,15]$. Singh et al. presented the first report of clinically and statistically significant improvement in pain and quality of life after a single injection of 100 units of BoNT-A in patients with moderate to severe refractory shoulder joint pain [6]. At 1 month post injection, the VAS pain reduction was significantly more in the BoNT-A group versus the placebo group ( -2.4 vs-0.8; Pvalue $=0.014)$. Boon et al. evaluated the efficacy and safety of BoNT-A injected intra-articularly in 60 subjects with moderate pain and functional impairment secondary to knee OA [14]. Subjects were randomized to receive a single injection of corticosteroid, low-dose BoNT-A (100 units), or high-dose BoNT-A (200 units). Only the lowdose BoNT-A group showed significantly decreased in pain VAS score from $6.6+1.9$ to $4.5+2.2(\mathrm{P}=0.01)$ at 8 weeks. In our study, the mean VAS for ankle pain reduced from $4.0 \pm 1.8$ to $1.8 \pm 0.9$ at 6 monthfollow-up $(\mathrm{P}<0.001)$. Although the described improvement regarding pain and disability was also documented in this present study, the results were difficult to compare because of different joint injections and different radiographic disease severity.

BoNT-A has been extensively studied and used clinically for its muscle paralyzing effects, but there is increasing evidence to support using it in pain modulation $[16,17]$. Recent pilot studies report that intra-articular injection of BoNT-A into painful joints of patients with various types of arthritis leads to significant and durable improvement in pain and function and is safe to use [4-6,18]. In previous small open label studies, initial effects for BoNT-A were encouraging because two thirds of the patients had more than $50 \%$ reduction in joint pain severity that was associated with a significant improvement in function [18]. Joint pain decreased within 2-14 days. Time to maximal pain relief varied from 4 to 12 weeks, and the effects lasted between 3 to 13 months [4].

The exact mode of action of BoNT-A in OA remains unknown. OA represents a complexity of pain conditions, including manifestations of both nociceptive and neuropathic mechanisms driven by joint pathophysiology and abnormal excitability in peripheral and central pain pathways [19-25]. The peripheral and central sensitization may amplify the joint pain. The mechanism of BoNT-A action is related to inhibiting transmitter release from nerve fibers [26,27]. BoNT-A binds to nociceptor C-fibers, undergoes endocytosis and blocks the vesicle release of agents involved in joint pain generation and transmission to the spinal cord. These agents also sensitize the nociceptor by neurogenic inflammation. More recent studies have also indicated a potential for inhibiting the release of mediators involved in nociception such as substance $\mathrm{P}$, calcitonin gene related peptide and glutamate, which leads to a decrease in pain transmission and peripheral sensitization [28].

Interestingly, we demonstrate that injecting BoNT-A into the ankle joints is associated with reduction in pain and disability, as well as improvement in balance function. Balance is an important component of performance for transfer, ambulatory tasks and many daily activities. The presence of $\mathrm{OA}$ may accelerate the deterioration of balance control systems. Reduced muscle strength and deficits in lower limb proprioception associated with OA can compromise effective and timely motor responses for maintaining balance [29,30]. Pain in OA may result in reduced loading of the affected joint, potentially jeopardizing an individual's ability to maintain balance. Although the mechanism by which BoNT-A results in a clinical improvement in balance remains unknown, we think that pain reduction may be a major contributing factor.

The results of our study support that BoNT-A injection may be safe and effective for the treatment of ankle OA. This current study is encouraging, as it continues to build on the existing data suggesting benefits from BoNT-A injections in the joints. The positive result of using BoNT-A in patients with ankle OA suggests that it is a promising approach worthy of serious clinical investigation[31].

The study had several limitations. The number of participants was relatively small and they were not blinded in treatment. The study did not include a control group, thus the results should be interpreted with caution, because some improvements might be the result of the placebo effect. We only recruited patients with Kellgren-Lawrence grade 2 ankle OA, the results cannot be generalized to other OA populations with different radiographic severity. Future studies, which include larger number of patients with more rigorous study controls are necessary to determine the efficacy of BoNT-A injections for ankle OA. The predictive factors for good response should be explored. The efficacy of BoNT-A compared to corticosteroid injections, different molecular weight hyaluronate, NSAIDs, or therapeutic exercise, as well as other potential combination therapy, may help determine the best overall treatment plan for patients with ankle OA.

\section{Conclusion}

Intra-articular BoNT-A injection to the ankle joint is associated with improvement in pain, function and balance in patients with painful ankle OA. These effects can last for 6 months. Future studies with larger double-blind randomized studies are needed for more definite conclusions.

\section{Acknowledgements}

We wish to express our sincere gratitude to all the investigators who participated in the trial. The study was supported by a grant of VGHKS100-061 (an academic research fund from the hospital's medical research council).

\section{References}

1. Mahowald M (2000) The role of nonprescription analgesics in treating mild to moderate pain. Clinical and economic considerations. Health Learning Systems, Minneapolis, USA. 
Citation: Liou IH, Sun SF, Hsu CW, Lin HS, Chou YJ, et al. (2014) Intraarticular Botulinum Toxin A for the Treatment of Painful Ankle Osteoarthritis-A Pilot Study. J Arthritis 3: 1000127. doi:10.4172/2167-7921.1000127

Page 5 of 5

2. Mahowald M (2004) Chronic Pain Management In: Ruddy S, Harris E, Sledge C, Budd R, Sergent J (Eds.) Kelley's Textbook of Rheumatology. WB Saunders, Philadelphia, PA.

3. American College of Rheumatology (2000) Recommendations for the medical management of osteoarthritis of the hip and knee, 2000 update. ACR Subcommittee on Osteoarthritis Guidelines. Arth. Rheum. 43: 1905-1915.

4. Mahowald ML, Singh JA, Dykstra D (2006) Long term effects of intraarticular botulinum toxin A for refractory joint pain. Neurotox Res 9: 179-188.

5. Singh JA, Mahowald ML, Kushnaryov A, Goelz E, Dykstra D (2009) Repeat injections of intra-articular botulinum toxin a for the treatment of chronic arthritis joint pain. J Clin Rheumatol 15: 35-38.

6. Singh JA, Mahowald ML, Noorbaloochi S (2009) Intra-articular botulinum toxin A for refractory shoulder pain: a randomized, doubleblinded, placebo-controlled trial. Transl Res 153: 205-216.

7. Dykstra DD, Stuckey MW, Schimpff SN, et al. (2007) The effects of intraarticular botulinum toxin on sacroiliac, cervical/lumbar facet and sternocalvicular joint pain and C-2 root and lumbar disc pain: a case series of 11 patients. The Pain Clinic 19: 27-32.

8. KELLGREN JH, LAWRENCE JS (1957) Radiological assessment of osteo-arthrosis. Ann Rheum Dis 16: 494-502.

9. Domsic RT, Saltzman CL (1998) Ankle osteoarthritis scale. Foot Ankle Int 19: 466-471.

10. Kitaoka HB, Alexander IJ, Adelaar RS, Nunley JA, Myerson MS, et al. (1994) Clinical rating systems for the ankle-hindfoot, midfoot, hallux, and lesser toes. Foot Ankle Int 15: 349-353.

11. Huskisson EC (1974) Measurement of pain. Lancet 2: 1127-1131.

12. Hurvitz EA, Richardson JK, Werner RA, Ruhl AM, Dixon MR (2000) Unipedal stance testing as an indicator of fall risk among older outpatients. Arch Phys Med Rehabil 81: 587-591.

13. Podsiadlo D, Richardson S (1991) The timed "Up \& Go": a test of basic functional mobility for frail elderly persons. J Am Geriatr Soc 39: 142-148.

14. Boon AJ, Smith J, Dahm DL, Sorenson EJ, Larson DR, et al. (2010) Efficacy of intra-articular botulinum toxin type $A$ in painful knee osteoarthritis: a pilot study. PM R 2: 268-276.

15. Chou CL, Lee SH, Lu SY, Tsai KL, Ho CY, et al. (2010) Therapeutic effects of intra-articular botulinum neurotoxin in advanced knee osteoarthritis. J Chin Med Assoc 73: 573-580.

16. Rowland LP (2002) Stroke, spasticity, and botulinum toxin. N Engl J Med 347: 382-383.
17. Mense S (2004) Neurobiological basis for the use of botulinum toxin in pain therapy. J Neurol 251 Suppl 1: I1-7.

18. Mahowald ML1, Krug HE, Singh JA, Dykstra D (2009) Intra-articular Botulinum Toxin Type A: a new approach to treat arthritis joint pain. Toxicon 54: 658-667.

19. Schaible HG, Del Rosso A, Matucci-Cerinic M (2005) Neurogenic aspects of inflammation. Rheum Dis Clin North Am 31: 77-101, ix.

20. Schaible HG, Schmelz M, Tegeder I (2006) Pathophysiology and treatment of pain in joint disease. Adv Drug Deliv Rev 58: 323-342.

21. Schaible HG, Richter F, Ebersberger A, Boettger MK, Vanegas H, et al. (2009) Joint pain. Exp Brain Res 196: 153-162.

22. Schaible HG (2007) Peripheral and central mechanisms of pain generation. Handb Exp Pharmacol : 3-28.

23. Konttinen YT, Kemppinen P, Segerberg M, Hukkanen M, Rees R, et al. (1994) Peripheral and spinal neural mechanisms in arthritis, with particular reference to treatment of inflammation and pain. Arthritis Rheum 37: 965-982.

24. Konttinen YT, Kemppinen P, Segerberg M, Hukkanen M, Rees R, et al. (1994) Peripheral and spinal neural mechanisms in arthritis, with particular reference to treatment of inflammation and pain. Arthritis Rheum 37: 965-982.

25. Garrett NE, Mapp PI, Cruwys SC, Kidd BL, Blake DR (1992) Role of substance P in inflammatory arthritis. Ann Rheum Dis 51: 1014-1018.

26. Welch MJ, Purkiss JR, Foster KA (2000) Sensitivity of embryonic rat dorsal root ganglia neurons to Clostridium botulinum neurotoxins. Toxicon 38: 245-258.

27. Aoki KR (2003) Evidence for antinociceptive activity of botulinum toxin type A in pain management. Headache 43 Suppl 1: S9-15.

28. Dolly O (2003) Synaptic transmission: inhibition of neurotransmitter release by botulinum toxins. Headache 43 Suppl 1: S16-24.

29. Durham PL, Cady R, Cady R (2004) Regulation of calcitonin gene-related peptide secretion from trigeminal nerve cells by botulinum toxin type A: implications for migraine therapy. Headache 44: 35-42.

30. Fisher NM, Pendergast DR (1997) Reduced muscle function in patients with osteoarthritis. Scand J Rehabil Med 29: 213-221.

31. Hassan BS, Mockett S, Doherty M (2001) Static postural sway, proprioception, and maximal voluntary quadriceps contraction in patients with knee osteoarthritis and normal control subjects. Ann Rheum Dis 60: 612-618. 